\title{
Dereplication of macrocyclic trichothecenes from extracts of filamentous fungi through UV and NMR profiles
}

\author{
Arlene A Sy-Cordero ${ }^{1}$, Tyler N Graf ${ }^{1}$, Mansukh C Wani $^{2}$, David J Kroll ${ }^{3}$, Cedric J Pearce ${ }^{4}$ \\ and Nicholas H Oberlies ${ }^{1}$
}

Macrocyclic trichothecenes (MTs), which have potent cytotoxicity, have been isolated from many different fungal species. These compounds were evaluated clinically by the US National Cancer Institute in the 1970s and 1980s. However, they have yet to be advanced into viable drugs because of severe side effects. Our team is investigating a diverse library of filamentous fungi for new anticancer leads. To avoid reisolating MTs through bioactivity-directed fractionation studies, a protocol for their facile dereplication was developed. The method uses readily available photodiode array detectors to identify one of two types of characteristic UV spectra for these compounds. In addition, diagnostic signals can be observed in the ${ }^{1} \mathrm{H}-\mathrm{NMR}$ spectra, particularly for the epoxide and conjugated diene moieties, even at the level of a crude extract. Using these techniques in a complementary manner, MTs can be dereplicated rapidly.

The Journal of Antibiotics (2010) 63, 539-544; doi:10.1038/ja.2010.77; published online 21 July 2010

Keywords: dereplication; filamentous fungi; macrocyclic trichothecenes

\section{INTRODUCTION}

Macrocyclic trichothecenes (MTs) are a class of sesquiterpene lactones that are reported to have a wide array of biological activities, including antimalarial, ${ }^{1-3}$ antileukemic, ${ }^{4}$ antibiotic, ${ }^{5}$ antifungal ${ }^{6}$ antiviral $^{7}$ and anticancer. ${ }^{8-11}$ According to Grove, ${ }^{12}$ they were first described in 1946 and isolated in pure form in 1962, and their potent biological activity and interesting chemistry stimulated a large amount of research throughout the 1970s and 1980s. Grove's comprehensive review ${ }^{12}$ of the literature on MTs through 1991 should be referenced for more complete details on the history, chemistry and structural variants of MTs, which at that time were noted at over 60 distinct compounds and today probably represent over 100 compounds. ${ }^{1,13}$ Moreover, Jarvis and Mazzola ${ }^{4}$ reviewed both the biological activity of these compounds, which are reported to effect cell growth through inhibition of protein synthesis, and their evaluation against in vivo models of cancer by the US National Cancer Institute (NCI). Jarvis et al. ${ }^{14,15}$ conducted medicinal chemistry studies on analogs of the MTs as well. Yet, despite evaluating over 90 natural and synthetic analogs of MTs in in vitro and in vivo models of cancer, only two MTs were evaluated clinically by the NCI, and both were dropped because of severe toxicity (Newman DJ, National Cancer Institute, Frederick, MD, USA, personal communication).
In the course of our early research on filamentous fungi, a project that targets the isolation of structurally unique compounds with potent anticancer activity, ${ }^{16,17}$ several MTs (for example, verrucarin A (1) and roridin E (2)) were isolated through bioactivity-directed fractionation based on cytotoxic activity vs a human tumor panel consisting of three cell lines. Not surprisingly, based on the literature, these MTs and related analogs were quite cytotoxic, being on the same order of potency as the positive control, camptothecin, even when the MTs were present at a low concentration in a crude extract. As such, the exciting cytotoxicity results skewed the chemistry efforts toward the isolation and elucidation of known MTs and/or closely related analogs, and in turn, pulled resources away from the discovery of bioactive leads with new structural types.

What was needed to circumvent this problem was a procedure to dereplicate MTs, particularly at the level of the crude extract and using techniques and tools that were readily available at the bench. As most of the filamentous fungi under examination for this project, sampled from a library of over 50000 isolates, were of unknown taxonomy at the time of initial evaluation, ${ }^{16}$ genera well known to produce MTs (for example, Fusarium spp., Myrothecium spp., Stachybotrys spp. and Trichothecium spp. $)^{4}$ could not be eliminated indiscriminately; other authors have noted similar challenges when relying on the taxonomy

${ }^{1}$ Department of Chemistry and Biochemistry, The University of North Carolina at Greensboro, Greensboro, NC, USA; ${ }^{2}$ Natural Products Laboratory, Research Triangle Institute, Research Triangle Park, NC, USA; ${ }^{3}$ Department of Pharmaceutical Sciences, BRITE, North Carolina Central University, Durham, NC, USA and ${ }^{4}$ Mycosynthetix Inc., Hillsborough, NC, USA

Correspondence: Dr NH Oberlies, Department of Chemistry and Biochemistry, The University of North Carolina at Greensboro, Greensboro, NC 27402, USA. E-mail: Nicholas_Oberlies@uncg.edu

Received 1 April 2010; revised 7 June 2010; accepted 8 June 2010; published online 21 July 2010 
of fungi for dereplication. ${ }^{18}$ Moreover, with three notable exceptions, ${ }^{13,18,19}$ the literature on analytical dereplication procedures for MTs was scant, probably because, as Grove observed, ${ }^{12}$ MTs have not been implicated as key mycotoxin contaminants of grain or grain products, and thus, there has not been a demand to develop analytical methods for their rapid identification/quantification. The exceptions were promising and comprehensive; however, two relied on techniques and tools that may not be available in all laboratories, namely HR-MS ${ }^{18}$ and capillary flow NMR. ${ }^{19}$ The third was more accessible, using photodiode array detection, although it focused on toxins of Stachybotrys sp. $;^{13}$ methods that could be applied to all structural variants of MTs were needed.

Thus, a dereplication strategy was developed and implemented that capitalized on the UV (through photodiode array detection) and ${ }^{1} \mathrm{H}$ NMR spectra. The procedure discerns readily samples that contain MTs, and the pattern recognition afforded by the UV spectra makes the strategy straightforward to implement.

\section{RESULTS AND DISCUSSION}

\section{Key structural features of MTs}

MTs are comprised of several structural features that are discernable in the ${ }^{1} \mathrm{H}$ NMR and/or UV spectra (Table 1). When coupled together, and supported by potent cytotoxicity, these features permitted the facile dereplication of even crude extracts for MTs. The most telling feature in the ${ }^{1} \mathrm{H}$ NMR spectrum may be the epoxide ring across positions $\mathrm{C}-12$ to $\mathrm{C}-13$, which is present in all but three MTs, ${ }^{12}$ and the cytotoxicity of this class of compounds was reported to decrease substantially when this moiety was absent. ${ }^{20}$ The two protons of the epoxide, $\mathrm{H}-13 \mathrm{a}$ and $\mathrm{H}-13 \mathrm{~b}$, displayed characteristic doublets $(J=4 \mathrm{~Hz})$ in the ${ }^{1} \mathrm{H}$ NMR spectrum at $\sim \delta 3.10$ and 2.78 . The sesquiterpene core of the molecule has a mono-substituted double bond across positions C-9 to C-10, and this rendered two more distinguishing features in the ${ }^{1} \mathrm{H}$ NMR spectrum. The only proton of this double bond, $\mathrm{H}-10$, displayed a broad doublet at $\sim \delta 5.45(J=6 \mathrm{~Hz})$. Moreover, the methyl substituent at position C-9 (that is, methyl C-16) displayed a singlet for $\mathrm{H}_{3}-16$ at $\sim \delta 1.73$; at higher field, allylic coupling of $\sim 1 \mathrm{~Hz}$ may be observed between $\mathrm{H}_{3}-16$ and $\mathrm{H}-10$. Finally, the macrocycle moiety, which loops from $\mathrm{C}-4$ to $\mathrm{C}-15$ through a carbon chain of variable lengths (typically $\mathrm{C}_{11}$ or $\mathrm{C}_{12}$ chains), includes $\alpha, \beta$-unsaturated lactone moieties that provided key spectroscopic features, particularly in UV spectra. ${ }^{18}$ In the case of compounds such as verrucarin A (1), there was a single UV maximum at $\sim 260 \mathrm{~nm}$. Whereas, in the case of compounds such as roridin E, the additional unsaturated carbonyl accounts for a second UV peak at $\sim 230 \mathrm{~nm}$. These dienes also produced key signals in the olefinic region of the ${ }^{1} \mathrm{H}$ NMR (that is, $\delta$ 5.72-8.01), but the UV spectrum provides a tool that is readily discernable. In summary, when an active extract was identified, if these key structural features were noted in the ${ }^{1} \mathrm{H}$ NMR data and/or the UV spectrum (Table 1), then the activity of the fungus was ascribed with great confidence to MTs.

\section{Development of the dereplication methodology}

Initial screening of 154 organic extracts of filamentous fungi for cytotoxicity against a human tumor panel resulted in the identification of eight active samples, two of which were the most potent. One of these two was carried through a bioactivity-directed fractionation. After several rounds of chromatography followed by assessment of cytotoxicity, the activity could be ascribed to two known compounds, the MTs verrucarin A (1) and roridin E (2), among other similar compounds; the ${ }^{1} \mathrm{H}$ and ${ }^{13} \mathrm{C}$ NMR data for $\mathbf{1}$ and $\mathbf{2}$ were in excellent agreement with the literature. 1,6,21,22 The taxonomy of the fungus (MSX 28737) was evaluated and found to be closely related to Myrothecium roridum, a known producer of MTs. ${ }^{23}$ However, at the time of initial examination, the taxonomy of the fungus was unknown.

The isolation of these two known MTs was somewhat disappointing, and the team feared that the potent activity of other extracts could be due to similar MTs. Thus, the MTs isolated from MSX 28737 were used as reference standards to develop the dereplication methodology. Figures 1 and 2 display the structures of compounds 1 and 2 and their corresponding UV and ${ }^{1} \mathrm{H}$ NMR spectra. Prominent signals in the NMR spectra (Figure 2) are notated as they relate to the key signals outlined in Table 1 . Specifically, the epoxide protons $\left(\mathrm{H}_{2}-13\right)$, the olefinic proton $(\mathrm{H}-10)$ and the vinylic methyl group $\left(\mathrm{H}_{3}-16\right)$ were observed in both compounds $\mathbf{1}$ and $\mathbf{2}$. If there were major differences between the two with respect to their ${ }^{1} \mathrm{H}$ NMR spectra, it was only the larger number of olefinic signals observed in compound 2. However, the key differences between the verrucarin-like compounds (based on 1) and the roridin-like compounds (based on 2) was discernable in the UV spectra (Figure 1). The macrocyclic portion of 1 resulted in a single UV maximum $(\sim 260 \mathrm{~nm})$, whereas the added unsaturation in the macrocycle of 2 resulted in two UV maxima ( $\sim 260$ and $230 \mathrm{~nm}$ ). In essence, these two pieces of spectroscopic data $\left({ }^{1} \mathrm{H}\right.$ NMR and UV), both of which can be acquired readily on relatively crude extracts, work in a complementary manner to dereplicate MTs. The ${ }^{1} \mathrm{H}$ NMR data identified key features of the sesquiterpene core (for example, epoxide, monosubstituted olefin, vinylic methyl group), whereas the UV data identified aspects of the macrocycle (for example, more or less unsaturation). In particular, the visual nature of the UV spectrum may be the most straightforward to recognize. For example, Figure 3 displays a prep-HPLC chromatogram of an active fraction, and several

Table 1 Key structural features and spectroscopic properties of macrocyclic trichothecenes

Structural feature
Epoxide across positions C-12 to C-13
Double bond across positions C-9 to C-10
Vinyl methyl group at position C-16
Two $\alpha, \beta$-unsaturated lactone moieties at positions C-6' to C-11' (for example,
compounds similar to verrucarin A (1))
Additional $\alpha, \beta$-unsaturated lactone moieties at positions $C-1^{\prime}$ to $C-3^{\prime}$
(for example, compounds similar to roridin E (2))

Spectroscopic properties

Two prominent doublets $(\mathrm{H}-13 \mathrm{a}$ and $\mathrm{H}-13 \mathrm{~b})$ in the ${ }^{1} \mathrm{H}$ NMR spectrum (for example, $\delta 3.10$ and $2.78 ; \mathrm{J}=4 \mathrm{~Hz}$ )

Prominent broad doublet (with possible allylic coupling at higher field) in ${ }^{1} \mathrm{H}$ NMR spectrum for $\mathrm{H}-10$ (for example, $\delta$ 5.45)

Prominent singlet (with possible allylic coupling at higher field) in ${ }^{1} \mathrm{H}$ NMR spectrum (for example, $\delta$ 1.73)

Prominent UV spectrum with a single maximum $(\lambda \sim 260 \mathrm{~nm})$

Prominent UV spectrum with two maxima ( $\lambda \sim 260$ and $230 \mathrm{~nm})$

(for example, compounds similar to roridin E (2)) 


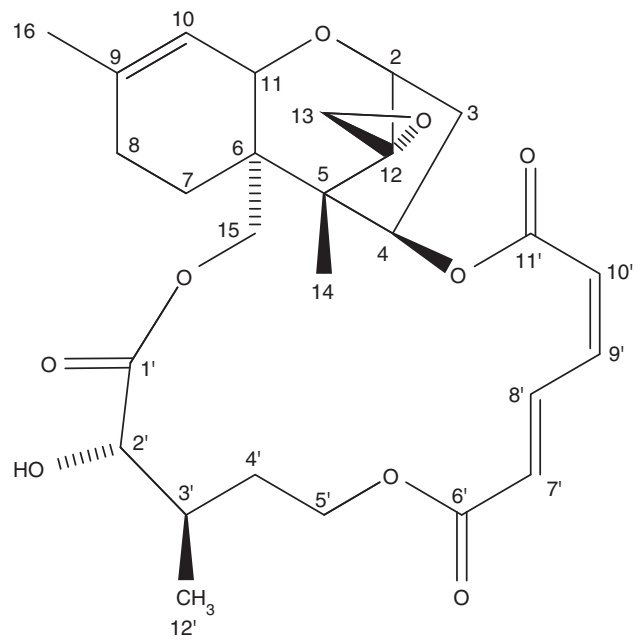

Verrucarin A (1)

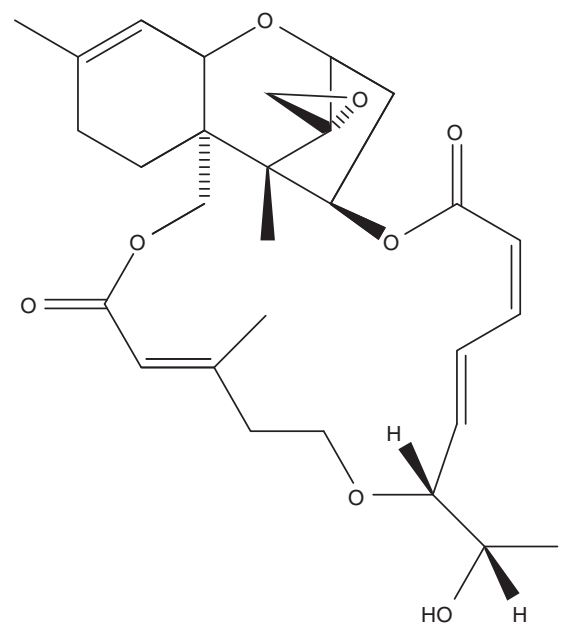

Roridin E (2)

Figure 1 Structures and UV profiles of verrucarin A (1) and roridin E (2).

peaks have a UV profile based on the roridin-like compounds, further justifying the dereplication of this sample.

\section{Use of the dereplication methodology}

Over the past year, this dereplication methodology has been used routinely on scores of cytotoxic extracts of filamentous fungi. For this, the goal was either (1) to determine that the cytotoxicity could be ascribed to MTs, in which case samples were dropped, or (2) to determine that the cytotoxicity cannot be ascribed to MTs, in which case samples were prioritized for bioactivity-directed fractionation.

The case of fungal strain MSX 15962 illustrates the use of these spectral techniques for dereplication (Table 1), especially when the taxonomy of the fungus did not suggest MTs. For example, based on the taxonomy of MSX 15962, which was found to be closely related to Gliocladium viride, one would not expect MTs. However, the ${ }^{1} \mathrm{H}$ NMR data (Figure 2) displayed some of the key signals of MTs. The methylene epoxide protons (encircled in Figure 2) were in the
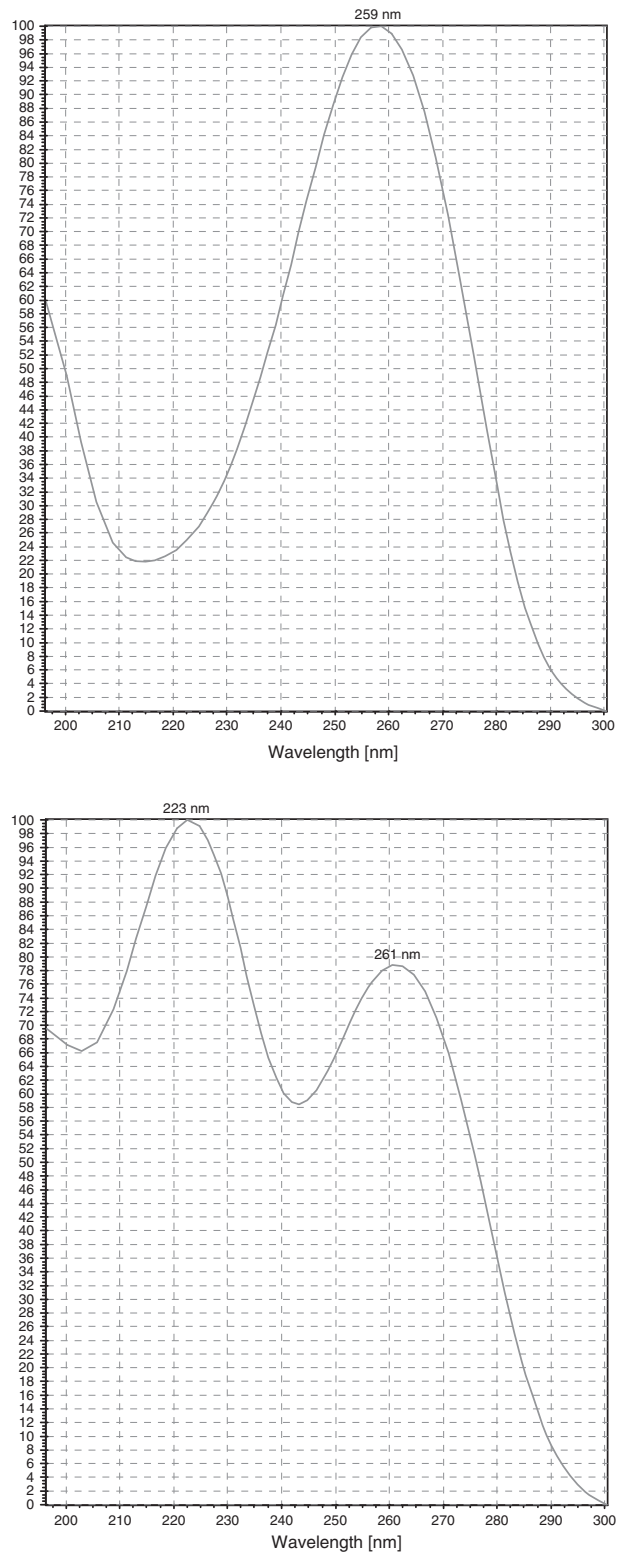

expected region, even when partially obscured at the level of the crude extract. Moreover, the downfield region (from $\sim 6$ to 8 p.p.m.; Figure 2) had signals typical of the MT olefinic protons. After initial rounds of bioactivity-directed fractionation, the activity concentrated in an HPLC fraction where the UV spectrum was diagnostic for roridin-type compounds (Figure 4). In short, the spectroscopic signatures of MTs, noted in both ${ }^{1} \mathrm{H}$ NMR and UV spectra of active fractions, permitted dereplication of this sample.

\section{Conclusion}

A method to dereplicate extracts of filamentous fungi for MTs was developed and implemented. The method used tools and instrumentation common to most laboratories of natural products, particularly the ${ }^{1} \mathrm{H}$ NMR and UV spectra. The pattern recognition afforded by these spectral techniques does not require sophisticated training of laboratory staff, and the methods were quite versatile, being applicable to crude extracts, partially purified mixtures, and pure compounds. 

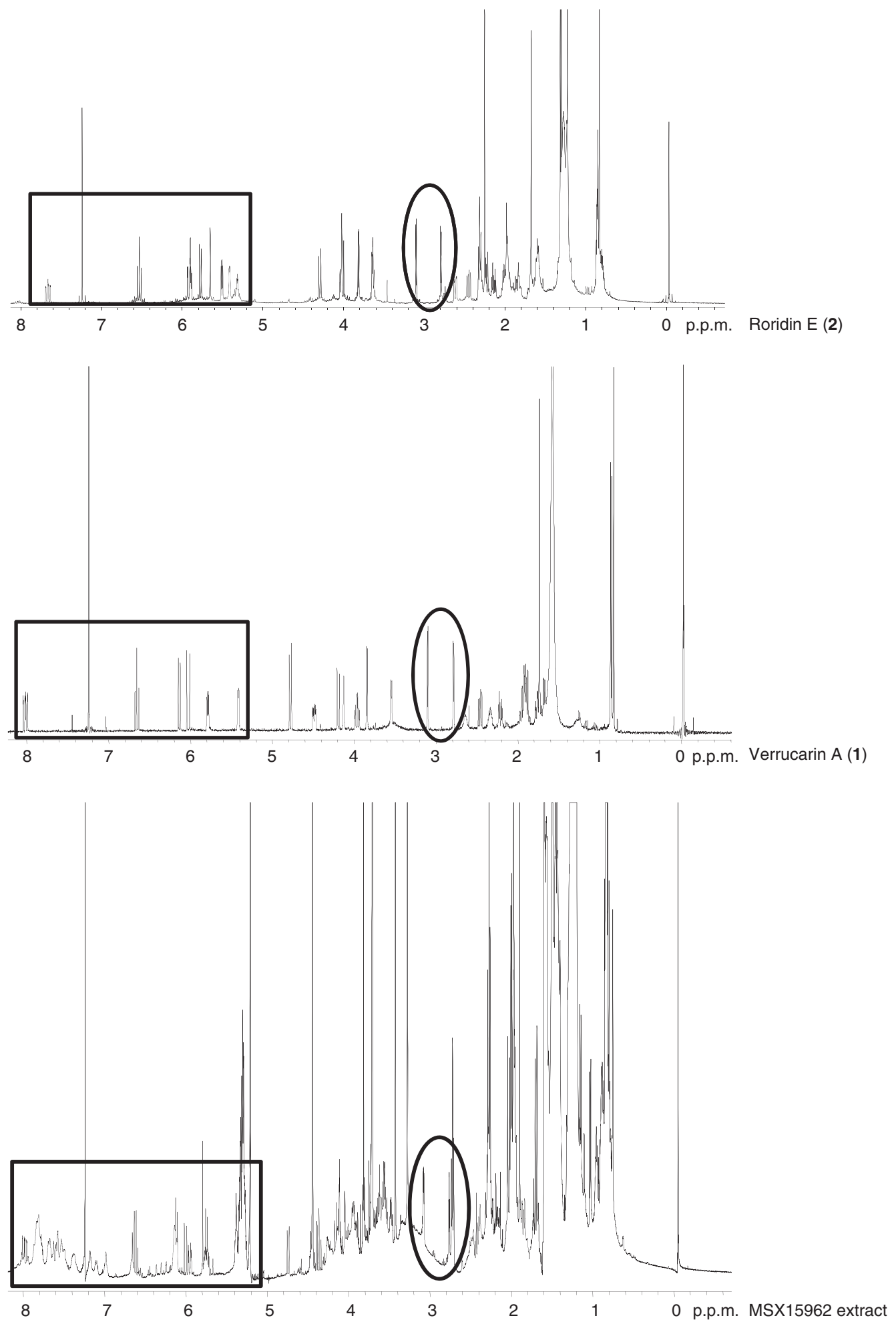

Figure $2{ }^{1} \mathrm{H}$ NMR spectra of the MTs, roridin E (2) and verrucarin A (1), and a crude extract that was dereplicated for MTs. The oval highlights the typical region for the chemical shifts of epoxide protons. The rectangle highlights the typical region for the chemical shift of olefinic protons.

\section{EXPERIMENTAL PROCEDURE}

\section{General}

All ${ }^{1} \mathrm{H}$ NMR experiments were performed in $\mathrm{CDCl}_{3}$ with TMS as an internal standard and were acquired on either a Varian Unity INOVA-500 (Varian Inc.,
Palo Alto, CA, USA) or a JEOL ECA-500 (JEOL USA, Inc., Peabody, MA, USA). Flash chromatography was conducted using a CombiFlash Rf system using RediSep Rf Si-gel columns (both from Teledyne-Isco; Lincoln, NE, USA). HPLC was carried out on a Prostar HPLC systems equipped with Prostar 210 pumps and a Prostar 335 photodiode array detector, with data collected and 

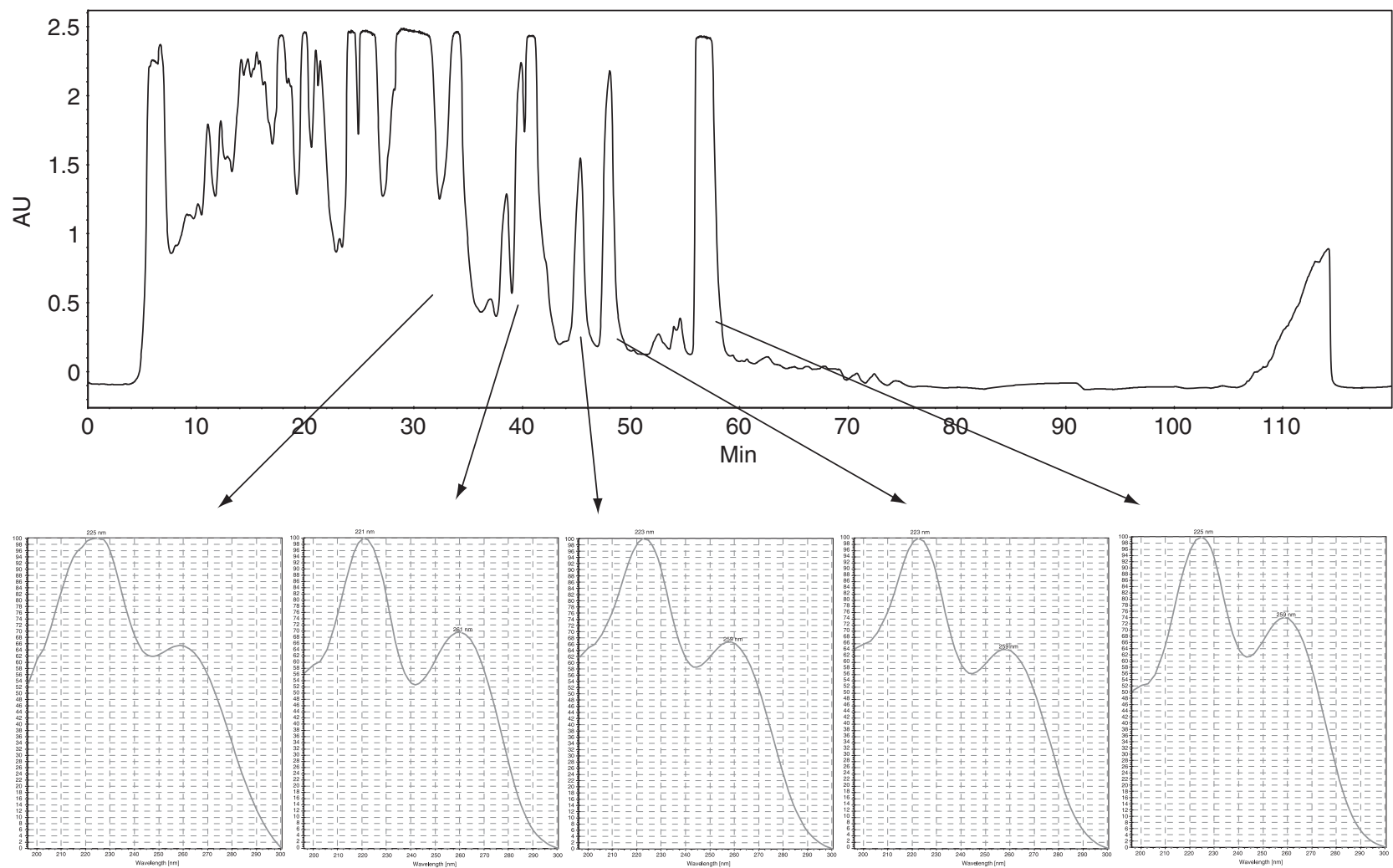

Figure 3 Prep-HPLC chromatogram (top) and UV spectra (bottom) of several potential MTs with a roridin-type macrocycle from another fraction of M. roridum (MSX 28737).
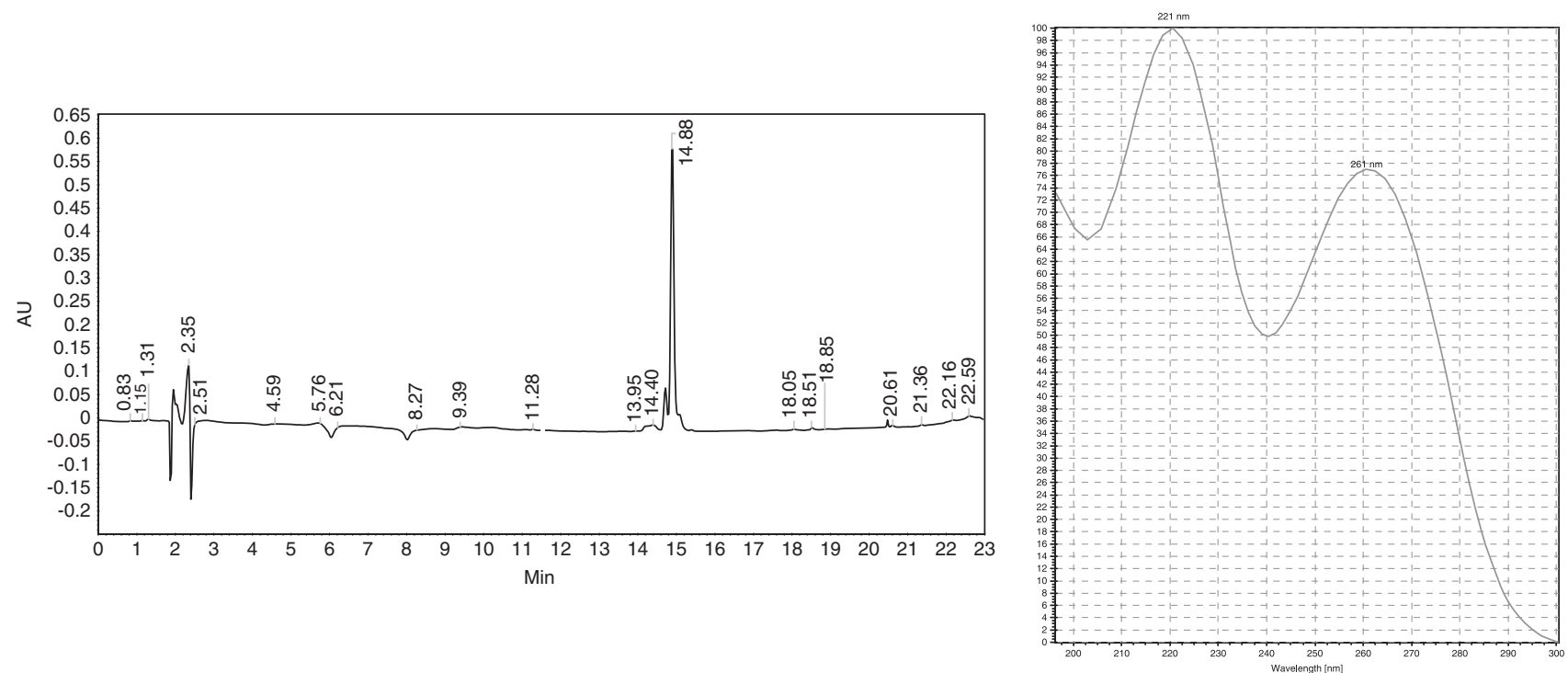

Figure 4 HPLC chromatogram (left) and UV spectrum (right) of an active fraction of MSX 15962.

analyzed using Galaxie Chromatography Workstation software (version 1.9.3.2) (All from Varian, Inc., Walnut Creek, CA, USA). For preparative HPLC, YMC ODS-A $(5 \mu \mathrm{m} ; 250 \times 20 \mathrm{~mm})$ columns were used with a $10 \mathrm{ml} \mathrm{min}^{-1}$ flow rate, whereas for analytical HPLC, YMC ODS-A $(5 \mu \mathrm{m} ; 150 \times 4.6 \mathrm{~mm})$ columns were used with a $1 \mathrm{ml} \mathrm{min}^{-1}$ flow rate (both from Waters; Milford, MA, USA). For analytical HPLC, MetaTherm HPLC column temperature controllers (Varian) maintained these columns at $30^{\circ} \mathrm{C}$.

\section{Producing organisms}

Both producing fungi were from the Mycosynthetix culture collection (Hillsborough, NC, USA). MSX 28737 was isolated from a sample of leaf litter, and the closest match using DNA analysis suggested it was M. roridum. MSX 15962 was isolated from aerial leaves in a varzea community from an area, which was occasionally flooded, and the closest match using DNA analysis suggested that it was G. viride. DNA analyses were performed by MIDI Labs (Newark, DE, 
USA), and the D2 variable region of the large subunit rRNA was sequenced and compared to the MIDI Labs database.

\section{Fermentations}

The cultures were grown on solid medium prepared using $12 \mathrm{~g}$ of rice to which was added $24 \mathrm{ml}$ of YESD medium prepared by adding yeast extract $(2 \% \mathrm{w} / \mathrm{v})$, soy peptone (2\%), dextrose $(2 \%)$ and malt extract $(1 \%)$ in water in $250 \mathrm{ml}$ Erlenmeyer flasks. This was sterilized at $121^{\circ} \mathrm{C}$ for $20 \mathrm{~min}$. For large-scale cultures, similar conditions were used, scaling to $150 \mathrm{~g}$ of rice and $300 \mathrm{ml}$ of YESD medium in 2.8-1 Fernback flasks. Cultures were inoculated either directly from a slant (small flasks), or for larger fermentations, by using an inoculum prepared by growing the fungus in a 50-ml polypropylene centrifuge tube containing $7 \mathrm{ml}$ of YESD medium incubated for 7 days at $22^{\circ} \mathrm{C}$ with agitation. The cultures were incubated at $22^{\circ} \mathrm{C}$ for $7-14$ days at which time they were extracted.

\section{Extraction and isolation}

A solid-phase fungal culture of MSX 28737 was scored with a spatula and submerged with $60 \mathrm{ml}$ of 1:1 $\mathrm{CHCl}_{3} / \mathrm{MeOH}$ and shaken overnight at 135 r.p.m. in a Lab-line Environ-shaker (Thermo Scientific, Waltham, MA, USA). The resultant solution was filtered on a Buchner funnel using Whatman no. 1 filter paper (Whatman International Ltd., Maidstone, UK), and $90 \mathrm{ml}$ of $\mathrm{CHCl}_{3}$ and $150 \mathrm{ml}$ of $\mathrm{H}_{2} \mathrm{O}$ were added to the filtrate to generate a biphasic solution of 4:1:5 $\left(\mathrm{CHCl}_{3}: \mathrm{MeOH}: \mathrm{H}_{2} \mathrm{O}\right)$. This solution was stirred for $2 \mathrm{~h}$, transferred to a separatory funnel, and the organic layer (lower) was removed. This organic extract was dried in vacuo $(127 \mathrm{mg})$ and then defatted by partitioning between hexane and $\mathrm{CH}_{3} \mathrm{CN}$. The $\mathrm{CH}_{3} \mathrm{CN}$ sample was dried $(41 \mathrm{mg})$ and subjected to reversed-phase HPLC (60:40 to 100:0 $\mathrm{CH}_{3} \mathrm{CN}: \mathrm{H}_{2} \mathrm{O}$ over $120 \mathrm{~min}$ ) to afford nine fractions. Fractions 3 and 5, both active in the cytotoxicity assay, were identified as verrucarin $\mathrm{A}(1,1 \mathrm{mg})$ and roridin $\mathrm{E}(2,1 \mathrm{mg})$; both compounds were $>96 \%$ pure by analytical reversed-phase HPLC.

A generalized methodology for dereplication of MTs begins by using essentially the same procedures outlined above, resulting in a defatted $\mathrm{CH}_{3} \mathrm{CN}$-soluble sample. This organic extract is then subjected to flash column chromatography using a RediSep Si-gel column and a two-stage gradient (100:0 to $0: 100$ hexane: $\mathrm{CHCl}_{3}$, then $100: 0$ to $75: 25 \mathrm{CHCl}_{3}: \mathrm{MeOH}$ ) over 70 column volumes total. Active pools from the initial column are then profiled by obtaining a ${ }^{1} \mathrm{H}$ NMR spectrum and performing analytical reversed-phase HPLC on a system equipped with a photodiode array detector.

\section{Cytotoxicity assays}

The cytotoxicity measurements were carried out as previously described ${ }^{24}$ and with the recently noted modification. ${ }^{25}$

\section{ACKNOWLEDGEMENTS}

This research was supported by P01 CA125066 from the National Cancer Institute/National Institutes of Health, Bethesda, MD, USA. The Golden LEAF Foundation (Rocky Mount, NC, USA) provided partial support to DJK. Mycology technical support was provided by Blaise Darveaux and Maurica Lawrence. We thank Drs AD Kinghorn and DS Ayers for helpful discussions.
1 Cole, R. J., Jarvis, B. B. \& Schweikert, M. A. Handbook of Secondary Fungal Metabolites xi, 672 pps (Academic Press, Amsterdam, Boston, 2003).

2 Zhang, H. J. et al. Antimalarial agents from plants. III. Trichothecenes from Ficus fistulosa and Rhaphidophora decursiva. Planta Med. 68, 1088-1091 (2002).

3 Isaka, M., Punya, J., Lertwerawat, Y., Tanticharoen, M. \& Thebtaranonth, Y. Antimalarial activity of macrocyclic trichothecenes isolated from the fungus Myrothecium verrucaria. J. Nat. Prod. 62, 329-331 (1999)

4 Jarvis, B. B. \& Mazzola, E. P. Macrocyclic and other novel trichothecenes-their structure, synthesis, and biological significance. Acc. Chem. Res. 15, 388-395 (1982).

5 Matsumoto, M., Minato, H., Uotani, N., Matsumoto, K. \& Kondo, E. New antibiotics from Cylindrocarpon sp. J. Antibiot. 30, 681-682 (1977).

6 Liu, J. Y. et al. Antifungal and new metabolites of Myrothecium sp. Z16, a fungus associated with white croaker Argyrosomus argentatus. J. Appl. Microbiol. 100, 195-202 (2006).

7 Garcia, C. C., Rosso, M. L., Bertoni, M. D., Maier, M. S. \& Damonte, E. B. Evaluation of the antiviral activity against Junin virus of macrocyclic trichothecenes produced by the hypocrealean epibiont of Baccharis coridifolia. Planta Med. 68, 209-212 (2002).

8 Bloem, R. J., Smitka, T. A., Bunge, R. H., French, J. C. \& Mazzola, E. P. Roridin-L-2, a new trichothecene. Tetrahedron Lett. 24, 249-252 (1983).

9 Smitka, T. A., Bunge, R. H., Bloem, R. J. \& French, J. C. Two new trichothecenes, PD 113,325 and PD 113,326. J. Antibiot. 37, 823-828 (1984).

10 Wagenaar, M. M. \& Clardy, J. Two new roridins isolated from Myrothecium sp. J. Antibiot. 54, 517-520 (2001).

11 Yu, N. J., Guo, S. X. \& Lu, H. Y. Cytotoxic macrocyclic trichothecenes from the mycelia of Calcarisporium arbuscula Preuss. J. Asian Nat. Prod. Res. 4, 179-183 (2002).

12 Grove, J. F. Macrocyclic trichothecenes. Nat. Prod. Rep. 10, 429-448 (1993).

13 Hinkley, S. F. \& Jarvis, B. B. Chromatographic method for Stachybotrys toxins. Methods Mol. Biol. 157, 173-194 (2001).

14 Jarvis, B. B., Midiwo, J. O. \& Mazzola, E. P. Antileukemic compounds derived by chemical modification of macrocyclic trichothecenes. 2. Derivatives of roridin-A and roridin-H and verrucarin-A and verrucarin-J. J. Med. Chem. 27, 239-244 (1984).

15 Jarvis, B. B., Stahly, G. P., Pavanasasivam, G. \& Mazzola, E. P. Anti-leukemic compounds derived from the chemical modification of macrocyclic trichothecenes. 1. Derivatives of verrucarin-A. J. Med. Chem. 23, 1054-1058 (1980).

16 Kinghorn, A. D. et al. Discovery of anticancer agents of diverse natural origin. Pure Appl. Chem. 81, 1051-1063 (2009).

17 Orjala, J., Oberlies, N. H., Pearce, C. J., Swanson, S. M. \& Kinghorn, A. D. Discovery of potential anticancer agents from aquatic cyanobacteria, filamentous fungi, and tropical plants. in Bioactive Compounds from Natural Sources 2nd edn, (ed Tringali, C.) (London, Taylor \& Francis: submitted).

18 Nielsen, K. F. \& Smedsgaard, J. Fungal metabolite screening: database of 474 mycotoxins and fungal metabolites for dereplication by standardised liquid chromatography-UV-mass spectrometry methodology. J. Chromatogr. A 1002, 111-136 (2003).

19 Lang, G. et al. Evolving trends in the dereplication of natural product extracts: new methodology for rapid, small-scale investigation of natural product extracts. J. Nat. Prod. 71, 1595-1599 (2008).

20 Grove, J. F. Non-macrocyclic trichothecenes. Nat. Prod. Rep. 5, 187-209 (1988).

21 Namikoshi, M. et al. A new macrocyclic trichothecene, 12,13-deoxyroridin E, produced by the marine-derived fungus Myrothecium roridum collected in Palau. J. Nat. Prod. 64, 396-398 (2001).

22 Saikawa, Y. et al. Toxic principles of a poisonous mushroom Podostroma cornu-damae. Tetrahedron 57, 8277-8281 (2001).

23 Gutzwiller, J. \& Tamm, C. Uber die struktur von verrucarin B. Verrucarine und roridine, 6. Helv. Chim. Acta. 48, 177-182 (1965).

24 Alali, F. Q. et al. New colchicinoids from a native Jordanian meadow saffron, Colchicum brachyphyllum: isolation of the first naturally occurring dextrorotary colchicinoid. J. Nat. Prod. 68, 173-178 (2005).

$25 \mathrm{Li}, \mathrm{C}$. et al. Bioactive constituents of the stem bark of Mitrephora glabra. J. Nat. Prod. 72, 1949-1953 (2009). 